\title{
SHERLOCK HOLMES Y LA ENSEÑANZA DE LA INVESTIGACION SOCIOEDUCATIVA
}

SHERLOCK HOLMES AND THE TEACHING OF SOCIO-EDUCATIONAL RESEARCH

\section{Volumen 13, Número 3}

Setiembre - Diciembre

pp. $1-20$

Este número se publicó el 30 de setiembre de 2013

Luis Carlos Morales Zúñiga

Revista indizada en REDALYC, $\underline{\text { SCIELO }}$

Revista distribuida en las bases de datos:

CATÁLOGO DE LATINDEX, IRESIE, CLASE, DIALNET, DOAJ, E-REVIST@S, SHERPA/ROMEO, QUALIS, MIAR

Revista registrada en los directorios:

ULRICH'S, REDIE, RINACE, OEI, MAESTROTECA, PREAL, CLASCO 


\title{
SHERLOCK HOLMES Y LA ENSEÑANZA DE LA INVESTIGACION SOCIOEDUCATIVA
}

\author{
SHERLOCK HOLMES AND THE TEACHING OF SOCIO-EDUCATIONAL RESEARCH
}

\section{Luis Carlos Morales Zúñiga ${ }^{1}$}

Resumen: En este artículo se exponen los resultados de la utilización de la novela Estudio en Escarlata como una estrategia didáctica que apoya los procesos de enseñanza y aprendizaje de la investigación socio-educativa. En la primera parte se describe la estrategia didáctica y su fundamentación teórica así como los elementos centrales del trabajo de campo realizado. En la segunda parte del artículo, se exponen los resultados de un cuestionario aplicado a los estudiantes que participaron de la experiencia, con el fin de observar, desde su punto de vista, las posibilidades que este recurso brinda para la enseñanza de la investigación socio-educativa.

Palabras clave: EDUCACION, ESTRATEGIA DIDÁCTICA, INVESTIGACIÓN, LITERATURA, SHERLOCK HOLMES, EDUCACION SUPERIOR, UNIVERSIDAD DE COSTA RICA

\begin{abstract}
This article is about the use of the novel A study in scarlet, by Arthur Conan Doyle such as a teaching strategy that supports the learning process of socio-educational research. First, it describes the didactical strategy and the theoretical background on which the strategy is supported, furthermore, it describes the main characteristics of the field experience in which the strategy was applied. In the second part of the article, it exposes the answers to a survey applied to students who participated in the experience. This survey was related to the possibilities of the strategy for the teaching and learning of socio-educational research.
\end{abstract}

Keywords: EDUCATION, DIDACTIC STRATEGY, RESEARCH, LITERATURE, SHERLOCK HOLMES, HIGHER EDUCATION, UNIVERSITY OF COSTA RICA

\footnotetext{
1 Profesor de la Escuela de Formación Docente de la Universidad de Costa Rica. Profesor de la Maestría en Ciencias de la Educación, con mención en Administración Educativa, de la Universidad de Costa Rica Magíster Scientiae en Sociología por la Maestría Centroamericana en Sociología con sede en la Universidad de Costa Rica (UCR). Licenciado en la Enseñanza de los Estudios Sociales y la Educación Cívica.
}

Dirección electrónica: luis23m@gmail.com

Artículo recibido: 13 de febrero, 2013

Aprobado: 19 de agosto, 2013 


\section{Introducción}

Lo social -decía Emile Durkheim (2001) - se explica por lo social. Esta tesis implica que la investigación social es un proceso de construcción de conocimiento sobre un segmento de la realidad de la cual somos necesariamente parte. El investigador social estudia y trata de hacer inteligible un fenómeno que ha incidido sobre su propia conciencia, o más aun, que ha estructurado su conciencia, racionalidad y valores, tal como lo indicaba también Lucien Goldman (1979).

Si bien este proceso de construcción de conocimiento puede ser desarrollado desde diversos paradigmas y puntos de vista así como desde distintas perspectivas teóricometodológicas, existe una lógica procedimental de la investigación social que es relativamente compartida, y que se expresa mediante un lenguaje común, compartido por la comunidad académica de las ciencias sociales, y que hace posible la construcción, acumulación y superación constante del conocimiento científico-social.

La aprehensión de ese lenguaje técnico, de esos procedimientos, pero sobre todo, de esa lógica de la investigación no siempre resulta ser un proceso de enseñanza-aprendizaje sencillo, esto por distintas razones: una de ellas tiene que ver con que el aprendizaje de la investigación social es más fructífero cuando se hace investigación, es decir, cuando se lleva a la práctica; otra razón, tiene que ver con el propio nivel de dificultad de la investigación social, el cual es variable en función de los conocimientos previos de los estudiantes y del nivel investigativo que se busca alcanzar.

Un problema particular del curso FD 0545 Investigación para el Mejoramiento del Aprendizaje, de la Escuela de Formación Docente de la Universidad de Costa Rica, en relación con la enseñanza de la investigación social y educativa, es que se trata de procesos de enseñanza de la investigación que se llevan a cabo con estudiantes que proceden de áreas diversas, por lo cual es común compartir el acto educativo con estudiantes de enseñanza de la Música, de las Ciencias Naturales, de los Estudios Sociales, de la Matemática, de las Lenguas Modernas, y de otras áreas de la docencia.

Esta característica del curso aumenta la diversidad de puntos de vista desde diferentes áreas del conocimiento, lo cual enriquece el trabajo del curso, al mismo tiempo que incrementa el nivel de dificultad para la enseñanza de la investigación socioeducativa pues, debido a las carreras de procedencia de los estudiantes, cada uno posee niveles 
diferenciados de conocimientos previos: algunos están poco familiarizados, o no lo están del todo, con conceptos y corrientes teórico-metodológicas de la investigación socioeducativa.

Lo anterior no solo dificulta el proceso de aprendizaje de la investigación sino que puede ocasionar que los términos, conceptos, autores, textos, y discusiones epistemológicas, teóricas o metodológicas de la investigación socioeducativa resulten soporíferos para algunos estudiantes, por ser discusiones que están poco relacionadas con sus carreras de procedencia y, por lo tanto, encajar menos en su red de conocimientos previos.

Ante esto, la obra de Arthur Conan Doyle, Estudio en escarlata (1956) -primera obra en la que aparece el célebre personaje Sherlock Holmes y su compañero de aventuras, el Doctor Watson-, podría constituir una herramienta didáctica para acercar a los estudiantes, amenamente, a través del placer de la literatura, a algunos conceptos fundamentales de la investigación, en general, y de la investigación socioeducativa, en particular. Por tanto, el objetivo fundamental de este escrito es sistematizar la estrategia didáctica basada en el análisis de la obra Estudio en escarlata (1956), como parte del proceso de enseñanzaaprendizaje de la investigación socioeducativa.

Este proceso ha sido llevado a cabo en dos grupos distintos del curso de FD 0545 Investigación para el mejoramiento del aprendizaje, de la Escuela de Formación Docente de la Universidad de Costa Rica, durante el segundo ciclo de 2011 y el primer ciclo de 2012. El curso está dirigido a todos los estudiantes de bachillerato en las carreras de Educación Secundaria, excepto los estudiantes de la carrera de Enseñanza de la Psicología, además, a estudiantes de las carreras de Educación Primaria y Educación Preescolar, por lo cual, convergen en el curso áreas del conocimiento sumamente diversas.

Cabe señalar también, que este ensayo no constituye un análisis de la obra como tal, lo cual ha sido y seguirá siendo tema para muchos otros trabajos en el campo de las humanidades. Tampoco se trata de un análisis comparativo entre ambos grupos, debido a que, como sus características son similares y la aplicación de la estrategia didáctica se llevó a cabo siguiendo los mismos procedimientos, las respuestas de los estudiantes se asumen en general, como una sola fuente de información, poniendo el énfasis en la estrategia y no en la dinámica de los grupos y sus particularidades. 


\section{Notas sobre el Estudio en Escarlata}

A pesar de que el origen de la novela policial o el relato detectivesco se encuentra en Estados Unidos, específicamente en la obra Los crímenes de la calle Morgue, de Edgar Allan Poe (1809-1849), es en Inglaterra donde el género se ha cultivado de una manera sobresaliente y prolija, sobre todo por autores como G.K. Chesterton, Wilkie Collins, y Arthur Conan Doyle (Borges, 1998).

Los autores de este género oscilan entre la creación literaria (ficción-arte) y la lógica de investigación científica (razón-ciencia), pues la novela policial a través de una historia ficticia, que es evidentemente manipulada por el autor, nos muestra el proceder racional y la lógica del pensamiento en sus personajes, quienes dotados de grandes capacidades analíticas, logran resolver los más diferenciados misterios. En otras palabras, el relato detectivesco logra mediante la ficción acercarnos al modo racional del proceder científico de una manera artística. J.L. Borges se ha referido ya al procedimiento de este género literario:

En 1841, un pobre hombre de genio, cuya obra escrita es tal vez inferior a la vasta influencia ejercida por ella en las diversas literaturas del mundo, Edgar Allan Poe, publicó en Philadelphia, Los crímenes de la calle Morgue, el primer cuento policial que registra la historia. Este relato fija las leyes esenciales del género: el crimen enigmático $y$, a primera vista insoluble, el investigador sedentario que lo descifra por medio de la imaginación y la lógica, el caso referido por un amigo personal y un tanto borroso del investigador. (Borges, 2011, p. 52)

En el Estudio en escarlata, Conan Doyle (1956) utiliza esas mismas leyes esenciales del género, e inaugura, además, lo que será la larga carrera de Sherlock Holmes, personaje que goza de una reputación probablemente mayor que la de su creador, pues tanto las obras de Conan Doyle en las que entra en acción el famoso detective, así como el gran número de filmes que retratan a Holmes, han tenido un efecto de consagración para este personaje.

Es en el Estudio en Escarlata donde Sherlock Holmes despliega su lógica de la deducción y la pone en práctica en la resolución del caso. Holmes, procede en la resolución de sus casos como un científico procedería en la resolución de un problema investigativo, en un paradigma deductivo. Holmes primero reflexiona el problema, segundo recolecta la evidencia empírica que le permita establecer premisas, a partir de las cuales construir una explicación lógica que finalmente corroborara en el campo. 
El racionalismo en Holmes se basa precisamente en la capacidad para establecer los límites del problema de investigación y la evidencia en la cual apoyar sus reflexiones. I. Copi (1984) ha señalado con acierto sobre el proceder de Holmes:

Toda investigación seria comienza con algún hecho o grupo de hechos cuyo carácter problemático atrae la atención del detective o del científico y con los cuales se inicia todo el proceso de la búsqueda. Habitualmente, los datos iniciales que constituyen el problema son demasiado escasos como para sugerir por sí mismos una explicación totalmente satisfactoria, pero pueden sugerir -al investigador competente- alguna hipótesis preliminar que lo conduzca a la búsqueda de hechos adicionales. Se espera que estos hechos adicionales sean pistas importantes para la solución final. El investigador inexperto o chapucero ignorará a todos, excepto a los más obvios de ellos; en cambio, el trabajador cuidadoso tratará de ser completo en su examen de los hechos adicionales a los que lo ha conducido su hipótesis preliminar. Holmes, por supuesto, era el más precavido y laborioso de los investigadores. (p.194)

En Estudio en Escarlata, cuando Sherlock se dirige a la escena del crimen, que es el primer paso, así como el más obvio, decide bajar del coche en que viajaba, 100 yardas antes del lugar donde aconteció el crimen, con tal de observar la mayor cantidad de detalles y evidencias posibles y obtener mayor cantidad de datos empíricos en los cuales apoyar sus razonamientos. Es por ello que I. Copi (1984) califica a Holmes como el investigador más precavido de los investigadores, del cual se pueden aprender diversos aspectos relacionados con el proceso de investigación, entendido como una indagación y problematización constante de la realidad.

Además de esto, la obra Estudio en Escarlata presenta una serie de elementos, relacionados con la lógica y los hábitos del investigador, que son realmente útiles para ilustrar un curso de investigación. Sobre esos elementos, vistos desde la perspectiva del curso de Investigación para el mejoramiento del aprendizaje, se ocupa este ensayo.

\section{Fundamentación de la estrategia didáctica}

El uso de la literatura como herramienta de enseñanza de distintos contenidos no es una novedad en sí misma. Existen algunos trabajos que recogen diversas experiencias sobre 
el uso de la literatura para el estudio de contenidos variados. Por ejemplo, Bonorino Ramírez (2011) expone una forma de utilización de la literatura para la enseñanza del derecho:

En este trabajo se exploran tres variantes para utilizar la literatura como herramienta didáctica para la enseñanza del derecho y la teoría jurídica. La primera, denominada "derecho en la literatura“. La segunda, llamada "derecho como literatura“, en la que se proponen analogías metodológicas entre el estudio del derecho y la teoría literaria. Y la tercera, a la que se denomina "derecho y literatura“, que propone establecer analogías parciales entre la actividad de interpretación que realizan los juristas y la que se lleva a cabo no directamente en textos literarios, sino en los trabajos teóricos que se realizan sobre ellos. Para poder ofrecer una imagen adecuada de las posibilidades didácticas de cada una de ellas se desarrollan tres ejemplos: la novela Billy Budd, marinero de Herman Melville, la Teoría del derecho de Ronald Dworkin, y el texto de Borges Pierre Menard, autor del Quijote. (p. 73)

El autor muestra en este documento cómo la literatura puede jugar un papel protagónico en el estudio y en la enseñanza-aprendizaje del derecho, específicamente en la teoría jurídica. Este trabajo de Bonorino constituye un antecedente y al mismo tiempo un fundamento teórico interesante para nuestro trabajo, pues se asemeja en el sentido de que, mientras Bonorino propone la variante de derecho en la literatura, en el presente trabajo estamos ante un intento de proponer una variante didáctica que podría ser considerada como investigación en la literatura, también como literatura en la investigación, pues se trata de que los estudiantes, mediante la utilización de un recurso literario, observen el método deductivo, abductivo e inductivo (Sierra Bravo, 1987) utilizado por Sherlock Holmes;asimismo, la forma como este personaje expone la resolución de un caso a través de una forma particular de razonamiento y de una serie de pasos investigativos -modus operandi del investigador-, se inserta en lo que podríamos denominar el paradigma racionalista (Kuhn, 2006).

Otra propuesta interesante es la obra de Cely Rodríguez y Moreno Lache (2006) en la cual se propone el uso de la literatura para la enseñanza de la geografía. En este trabajo los autores plantean una propuesta didáctica que utiliza la obra El esquimal y la mariposa, del autor Nahum Montt, con el fin de profundizar en el estudio del espacio geográfico urbano. 
Es observable el interés por incorporar la literatura como una estrategia didáctica que mejore el estudio de algunos contenidos específicos en distintas disciplinas. Incluso en las ciencias médicas, el autor Josep E. Banos (2003) propone que la literatura es una herramienta muy útil para el estudio de la ética médica y de la relación médico-paciente:

El objetivo principal sería permitir la discusión de algunos aspectos de la actividad médica que considero primordiales para ejercer la profesión correctamente. Para alcanzarlo, se pretende que los estudiantes de los años preclínicos reconozcan pronto los elementos psicológicos y sociológicos asociados a la enfermedad, en el convencimiento que con ello se podría conseguir una actitud más positiva $\mathrm{y}$, más adelante, una práctica más humana de la medicina. Se desea, en fin, que los estudiantes consideren a los pacientes como personas que enferman y no como meros casos (p.10)

Como se puede apreciar en los tres documentos reseñados, el interés es doble: Por un lado, interesa abordar algunos contenidos o temas específicos (teoría jurídica, geografía urbana y ética de la medicina); por otro lado, hay un interés por humanizar la enseñanza de contenidos que, por la naturaleza de la disciplina, podrían presentarse como hechos científicos o académicos en sentido lato.

En el caso de la estrategia didáctica que presentamos, uno de los objetivos es apoyar el abordaje y el estudio de algunos contenidos específicos de la enseñanza de la investigación a partir novela Estudio en escarlata, tales contenidos son los siguientes:

1. Proceso de investigación

2. Tipos de razonamiento (deductivo - inductivo - abductivo)

3. Paradigmas científicos

4. Enfoques investigativos

5. Metodología de la investigación

6. Nociones de epistemología

7. Construcción del objeto de estudio

8. Técnicas de investigación

9. Hábitos del investigador

10. Gusto por la investigación 
Estos temas, entre otros, son estudiados en el curso Investigación para el Mejoramiento del Aprendizaje. La mayoría, excepto los temas nueve y diez, puede ser estudiada con una bibliografía estrictamente académica que se caracteriza por ser exhaustiva y amplia. Los dos temas que exceptuamos no se abordan normalmente en la literatura académica sobre investigación, pues se tienen por hechos dados o se pasan por alto (Eco, 1993).

En esa búsqueda por generar un gusto por la investigación, la novela Estudio en escarlata puede contribuir sobremanera, pues muestra de forma muy amena e interesante una manera de hacer investigación y de razonar lógicamente, lo cual es extrapolable al ámbito académico. Esto permite a los estudiantes, aprender conceptos, y elementos sobre la investigación social y educativa, sobre la lógica de la investigación, además de servir como un complemento placentero a los textos académicos que se estudian en el curso, pues permite experimentar el goce estético de la literatura.

\section{Descripción de la estrategia didáctica}

La estrategia didáctica propuesta en este escrito podría considerarse a partir de las siguientes fases:

1- La obra, el autor y el contexto: el docente realiza, en la primera sesión de trabajo, una reseña de generalidades de la obra y del contexto en que fue escrita, y una breve reseña biográfica de su autor Arthur Conan Doyle.

2- Para cada sesión de clase los estudiantes deben leer dos capítulos de la obra, y presentar un breve reporte escrito que incluye:

a. Una breve reseña de cada capítulo.

b. Un comentario sobre cuatro o más ideas del texto que se relacionan con la lógica de la investigación o con los contenidos del curso estudiados.

3- En cada sesión, un estudiante es responsable de iniciar el comentario de las ideas que le llamaron la atención, con el objetivo de empezar el diálogo y la retroalimentación.

4- Papel del docente:

a. Debe explicitar la importancia de la obra, del autor y del contexto en que fue escrita.

b. Dirigir y propiciar la discusión en la etapa de retroalimentación.

c. Aportar una lectura crítica e interpretativa del texto en relación con la investigación educativa. 
5- El trabajo está pensado para siete sesiones, pues son catorce capítulos, y se inicia durante la segunda mitad del curso, con el propósito de que los estudiantes puedan tener un marco referencial de la investigación que les permita una clave de lectura del texto.

6- El total de los reportes podría tener un valor sumativo en la nota final del curso.

Además de lo anterior, es importante señalar que, para profundizar en aspectos de la obra, han sido utilizados en la experiencia de campo algunos recursos que el contexto educativo en el que trabajamos nos permite. En este sentido, como apoyo, lo cual implica que no es imprescindible, han sido utilizados algunos recursos como videos, con el fin de ahondar en el contexto en que fue escrito la obra, el autor y en las características generales de los personajes principales: Sherlock Holmes y el Dr. Watson.

Sobre esto es preciso tener en cuenta que la presentación del carácter de los personajes puede ser un punto que se trate de manera muy general, para no crear en los estudiantes una especie de imagen previa de ellos que les condicione su descubrimiento y construcción, lo cual, como lectores, podrían realizar libremente.

Al finalizar la puesta en práctica de la estrategia didáctica, se aplica un cuestionario que incluye preguntas cerradas y abiertas, en el cual se busca recabar información sobre los contenidos del curso que se favorecen con el estudio y análisis de Estudio en Escarlata. Este cuestionario también recolecta información sobre las actividades que se pusieron en práctica durante el desarrollo de la estrategia didáctica, con el fin de evaluar la su eficacia, además, tomar en cuenta las sugerencias de los estudiantes para mejorar la estrategia.

\section{Aplicación de la estrategia y análisis de resultados}

La estrategia didáctica ha sido aplicada en el grupo 001 del curso FD 0545, Investigación para el Mejoramiento del Aprendizaje, en el segundo ciclo lectivo de 2011 y el primer ciclo lectivo de 2012. Tal como se señaló en la descripción de la estrategia didáctica, fueron utilizadas siete sesiones en las cuales trabajamos dos capítulos por sesión, cada capítulo estuvo a cargo de un estudiante, quien tenía la asignación de iniciar los comentarios y la discusión sobre los temas de la novela y su relación con la investigación socio-educativa. Después de la aplicación de la estrategia didáctica, al terminar la sétima sesión de las clases programadas para la actividad, cada estudiante completó un cuestionario, este tenía la 
finalidad de obtener la valoración de la estrategia desde la perspectiva de los participantes. La distribución de los estudiantes por carrera de procedencia es la siguiente:

Cuadro N. 1

\begin{tabular}{lc}
\hline \multicolumn{2}{c}{ Estudiantes según carrera de procedencia } \\
\hline \multicolumn{1}{c}{ Carrera } & $\begin{array}{c}\text { Número de } \\
\text { estudiantes }\end{array}$ \\
\hline Enseñanza de la Matemática & 5 \\
Enseñanza de las Artes Industriales & 1 \\
Enseñanza de las Ciencias Naturales & 4 \\
Enseñanza de los Estudios Sociales & 8 \\
Enseñanza del Castellano y Literatura & 9 \\
Enseñanza del Francés & 2 \\
Enseñanza del Inglés & 5 \\
Enseñanza de la Filosofía & 3 \\
Educación Primaria & 3 \\
Enseñanza de la Música & 5 \\
\hline \multicolumn{2}{c}{ Total } \\
\hline
\end{tabular}

Fuente: Elaboración propia a partir de la matrícula del curso FD 0135, grupo 001, II Ciclo 2011 y grupo 002 I Ciclo 2012.

Tal como se puede apreciar en el cuadro N. 1, la procedencia de los estudiantes que conforman el curso es sumamente variada: diez carreras diferentes. Esto implica formas diferentes de concebir la investigación, en algunos casos más o menos coincidentes, e igualmente, con avances diferenciados, lo cual hace que la experiencia en el curso pueda ser al mismo tiempo enriquecedora debido a la formación académica y profesional (incluyendo la experiencia personal de cada estudiante que conforma el curso) y compleja por el desarrollo diferenciado en la formación de cada estudiante.

Precisamente, el hecho de que el curso estuviese compuesto por estudiantes de tan diversa procedencia permitió un intercambio enriquecedor en el momento de discutir las impresiones del texto y de los incidentes que Sherlock Holmes y el Dr. Watson enfrentan en cada capítulo. Además, al tener múltiples lecturas de la obra, se establecieron distintas relaciones con la investigación socioeducativa, que a veces los demás participantes no habíamos observado, dándole un carácter más completo al análisis de la novela y de sus contenidos.

Como observador participante de la actividad, es conveniente resaltar que, en muchas sesiones de clase, la dinámica de comentar el texto literario hizo que las clases se 
"descargaran" por un momento del peso de las reflexiones y del trabajo académico y permitió un espacio para seguir pensando en los contenidos del curso, pero disfrutando de la literatura, lo cual resultó grato.

En el cuadro N. 2 se transcriben las consideraciones de los estudiantes en torno a la utilidad de la novela Estudio en escarlata como apoyo para el estudio de la investigación socioeducativa, de inmediato se describen las respuestas brindadas a cada una de las preguntas del cuestionario. $^{2}$

Cuadro N. 2

¿Considera que la novela Estudio en escarlata es útil como apoyo para el estudio de la investigación socio-educativa?

\begin{tabular}{|c|c|}
\hline Sí & No \\
\hline $\begin{array}{l}\text { - En la novela se evidencia cómo Holmes utiliza varios métodos investigativos } \\
\text { dando bases que se pueden utilizar en la investigación educativa, por ejemplo, la } \\
\text { deducción. } \\
\text { - Invita al receptor a sacar hipótesis, conclusiones, razona los casos. } \\
\text { - Se basa en procesos investigativos de donde se pueden extraer ejemplos de los } \\
\text { temas de investigación socioeducativa. } \\
\text { - Es una novela de fácil comprensión donde se percibe la investigación como un } \\
\text { proceso. } \\
\text { - Muestra los pasos que se deben seguir en el desarrollo de una investigación. } \\
\text { - Trae muy definidos los conceptos y una posible metodología de investigación. } \\
\text { - Tiene varias situaciones en las cuales se ven representados conceptos estudiados } \\
\text { - } \quad \text { Ayuda al estudiante a adentrarse al estudio de la investigación con el elemento } \\
\text { literario. } \\
\text { - Presenta pautas sobre cómo realizar una investigación. } \\
\text { - Es una novela entretenida que logra distraer de las lecturas "normales" del curso y } \\
\text { le pone más interés a la investigación. }\end{array}$ & $\begin{array}{l}\text { No hay } \\
\text { respuestas }\end{array}$ \\
\hline
\end{tabular}

Fuente: Elaboración propia a partir de cuestionario aplicado a los estudiantes del curso FD 0135, grupo 001, II ciclo 2011 y grupo 002, I ciclo 2012.

El total de los estudiantes contestó que sí considera la novela en cuestión como una herramienta útil de apoyo para el estudio de la investigación socioeducativa. Tal como se puede apreciar en el cuadro, no hay respuestas negativas y las razones por las cuales la novela se considera útil son variadas, pero básicamente se podrían resumir diciendo que, según los estudiantes, es una novela de fácil comprensión, que muestra de forma clara, aspectos relacionados con métodos investigativos, razonamiento deductivo, la investigación

${ }^{2}$ Se presenta en cada uno de los cuadros, las preguntas del cuestionario aplicado y las respuestas de los estudiantes, excluyendo aquellas respuestas que repiten ideas expresadas en varias ocasiones. 
concebida como un proceso y los pasos mediante los cuales el investigador puede ir desarrollando su trabajo.

Otro elemento destacable de los datos aportados por los estudiantes, en relación con la pregunta anterior, es que la utilización de la novela significó para ellos un espacio diferente al trabajo académico habitual del curso; al mismo tiempo, les permitió seguir pensando en la investigación de una forma diferente, tal como lo dicen en una de sus respuestas, incluyendo el elemento literario, lo cual, indican, le adhiere mayor interés al estudio de los elementos de investigación desarrollados en el curso.

Además de indagar sobre las impresiones de los estudiantes en torno a la utilización de la novela, el instrumento aplicado buscaba captar la perspectiva que ellos poseen sobre la metodología utilizada para el trabajo en clase a partir del texto, para lo cual se les preguntó si la metodología desarrollada en el curso es apropiada o no y cómo justifican su impresión al respecto.

En esta pregunta, tampoco hubo respuestas negativas, lo cual aporta evidencia y permite considerar que la perspectiva de los estudiantes en relación con la estrategia didáctica es muy favorable y que, en efecto, les permitió alcanzar un punto de vista diferente sobre la investigación socioeducativa, y sobre la investigación en general. A continuación se observan, en el cuadro N. 3, la pregunta y las respuestas de los estudiantes sobre el tema de la metodología desarrollada en el curso para el trabajo con la novela:

\section{Cuadro N. 3}


- A pesar de que es un reporte semanal no es tedioso y poder expresar nuestras opiniones personales lo hace más ameno.

- El trabajo de retroalimentación implementado por el docente es pertinente. Permite contrastar el texto y las nociones percibidas por el estudiante.

- Poco a poco se fue analizando la obra para ir descubriendo partes que ofrecían elementos de investigación.

Fuente: Elaboración propia a partir de cuestionario aplicado a los estudiantes del curso FD 0135, grupo 001, II ciclo 2011 y grupo 002, I ciclo 2012.

Como se puede notar en el cuadro anterior, hay distintas razones que dan cuenta de la impresión favorable que tienen los estudiantes en relación con la metodología utilizada en el marco de la estrategia didáctica aplicada. Sin embargo, esas razones podrían estar relacionadas con tres elementos principales, a saber, la retroalimentación y el intercambio de los distintos criterios de los estudiantes, las relaciones de los contenidos del curso con distintos aspectos de la novela y el hecho de dividir los reportes y el análisis de la obra por capítulos, lo cual, desde su punto de vista, les permite "ir descubriendo partes que ofrecían elementos de investigación".

En relación con el primer elemento, el cual hace de la estrategia didáctica un proceso constructivista de aprendizaje (Rosas; Sebastián, 2008) se podría indicar que el tema de la retroalimentación y el intercambio de ideas y de lecturas distintas de la obra, es un valor agregado al curso, pues permite plantear muy distintas interpretaciones del texto, lo cual se enriquece aún más, dadas las características del grupo, pues intervienen estudiantes de distintas carreras y formaciones, lo cual les da distintas posibilidades de lectura y de interpretación, ampliando colectivamente el análisis de la obra.

El segundo elemento evidencia que se ha cumplido con uno de los propósitos de la estrategia didáctica, el cual tiene que ver con que los estudiantes puedan observar la investigación en la literatura, como una forma diferente y grata de aprender sobre elementos básicos de investigación, al mismo tiempo, abrir un espacio en el cual, de una forma alternativa a la académica, los estudiantes se puedan acercar a los contenidos del curso, para ir descubriendo, de acuerdo con ellos, esos elementos de investigación que ofrece la novela y que, según la tercera categoría, se realiza de una forma práctica y gradual, al dividir el trabajo por capítulos.

En el cuadro N. 4, se puede apreciar lo que los estudiantes consideran como ventajas y limitaciones de la estrategia didáctica implementada, con el fin de obtener una perspectiva más amplia sobre el punto de vista de los estudiantes: 
Cuadro N. 4

Ventajas y limitaciones de las utilización de la novela Estudio en escarlata como apoyo para el estudio de la investigación socioeducativa

\begin{tabular}{|c|c|}
\hline Ventajas & Limitaciones \\
\hline $\begin{array}{l}\text { - Considero que es entretenido y permite dar } \\
\text { opiniones sobre técnicas de investigación. } \\
\text { - } \text { Diferente a otros cursos donde se limita a } \\
\text { tomar comprobaciones de lecturas. } \\
\text { - Se hace un poco más fácil comprender el } \\
\text { proceso investigativo. } \\
\text { - Es un texto interesante que promueve el } \\
\text { espíritu de un investigador, analítico y } \\
\text { reflexivo. } \\
\text { - Trabajar la lectura en clase aclara ideas } \\
\text { sobre el proceso de investigación. } \\
\text { - La novela es corta, entretenida, y la división } \\
\text { en capítulos facilita el trabajo semanal. } \\
\text { - Sigue el método científico explicado paso a } \\
\text { paso. } \\
\text { - La trama de la novela está muy ligada al } \\
\text { proceso investigativo. } \\
\text { - Obliga a encontrar detalles en la lectura que } \\
\text { normalmente no habría encontrado y a } \\
\text { relacionarlos con lo visto en el curso. } \\
\text { - Se muestra el uso del razonamiento } \\
\text { inductivo-deductivo. } \\
\text { - Se involucra al estudiante en el tema de la } \\
\text { investigación de una manera diferente y } \\
\text { agradable. } \\
\text { - Preparar los reportes ayuda a hacer una } \\
\text { reflexión sobre lo que estamos leyendo, o } \\
\text { sea, no es una lectura meramente pasiva. }\end{array}$ & $\begin{array}{l}\text { - Quizá el tiempo de lectura más las otras } \\
\text { lecturas propias del curso es complicado. } \\
\text { - Los informes no garantizan la apropiación del } \\
\text { conocimiento dado en la obra. } \\
\text { - Las traducciones de la novela no siempre } \\
\text { coinciden. } \\
\text { - Como se enfoca en lo investigativo se pierde } \\
\text { un poco la atención sobre la riqueza } \\
\text { narrativa. } \\
\text { - La diferencia de contextos. } \\
\text { - Que en la novela la investigación es empírica } \\
\text { y no académica, y si no se aclara podría } \\
\text { generar alguna confusión. } \\
\text { - Algunas personas participaron muy poco en } \\
\text { las conversaciones. }\end{array}$ \\
\hline
\end{tabular}

Fuente: Elaboración propia a partir de cuestionario aplicado a los estudiantes del curso FD 0135, grupo 001, II ciclo 2011 y grupo 002, I ciclo 2012.

Entre las ventajas que los estudiantes plantean, se reiteran algunos elementos que habíamos conocido ya en el Cuadro N. 3, los cuales tienen que ver con la posibilidad del intercambio y retroalimentación en clase, la relación de la novela con los contenidos del curso, precisamente con cuestiones de razonamiento inductivo y deductivo, el proceso y la metodología de investigación, el proceso de investigación, los paradigmas científicos, los enfoques investigativos, las nociones de epistemología, la construcción del objeto de estudio, las técnicas de investigación, los hábitos del investigador y el gusto por la investigación.

Además, en este cuadro se pueden apreciar algunos elementos más que los estudiantes consideran como ventajas: propiciar la reflexión sobre lo que se está leyendo y trabajando en el curso; favorecer la lectura activa, tanto de la obra como de las lecturas 
propias del curso, pues es necesario establecer relaciones; como plantea una de las respuestas, obliga[r] a encontrar detalles en la lectura que normalmente no habría encontrado y a relacionarlos con lo visto en el curso. Por tanto, esto nos permite deducir que la utilización de la novela tiene distintas ventajas que favorecen el aprendizaje de los contenidos del curso, de una manera alternativa al trabajo académico habitual.

Esta posibilidad de un acercamiento alternativo a los elementos de la investigación nos conduce a las limitaciones de la estrategia didáctica que plantean los estudiantes, pues, entre ellas, se indica, con toda razón, que la investigación que se realiza en la novela no es académica, sino empírica, lo cual podría generar confusión en los estudiantes; precisamente ahí, la intervención mediadora del docente y la capacidad de relación y creatividad de los estudiantes podría solventar esa particularidad de la novela y de la estrategia.

En cuanto a las otras limitaciones, hay dos que son inherentes al texto y a la obra: una tiene que ver con el problema de las traducciones, las cuales nunca coinciden con el original ni entre sí. El que una obra coincida absolutamente con el original es imposible, lo que sí es posible es tener una única y buena traducción de la obra, con tal de acercarnos lo más posible al original y que todos lean la misma traducción, tal como se realizó en esta experiencia.

Las otras limitaciones tienen que ver con el nivel de compromiso personal de cada estudiante en relación con la estrategia y sobre todo con su propia formación, pues se indica que los reportes no garantizan la apropiación de la obra, la lectura del texto más las lecturas del curso es complicado y algunos estudiantes no participaron tanto del intercambio. Como se puede apreciar, son elementos que dependen mucho de la personalidad y del compromiso de cada estudiante, aunque el docente puede intervenir tratando de propiciar la participación de todos los estudiantes, con la sensibilidad y el respeto hacia las distintas personalidades y formas de participación, pues la participación verbal no necesariamente es garantía de una participación significativa y viceversa.

Finalmente, con el instrumento se buscó captar la perspectiva de los estudiantes sobre cuáles contenidos propios del curso, fueron reforzados con la estrategia didáctica, se obtuvieron, como resultado, los siguientes datos: 


\section{Gráfico N. 1}

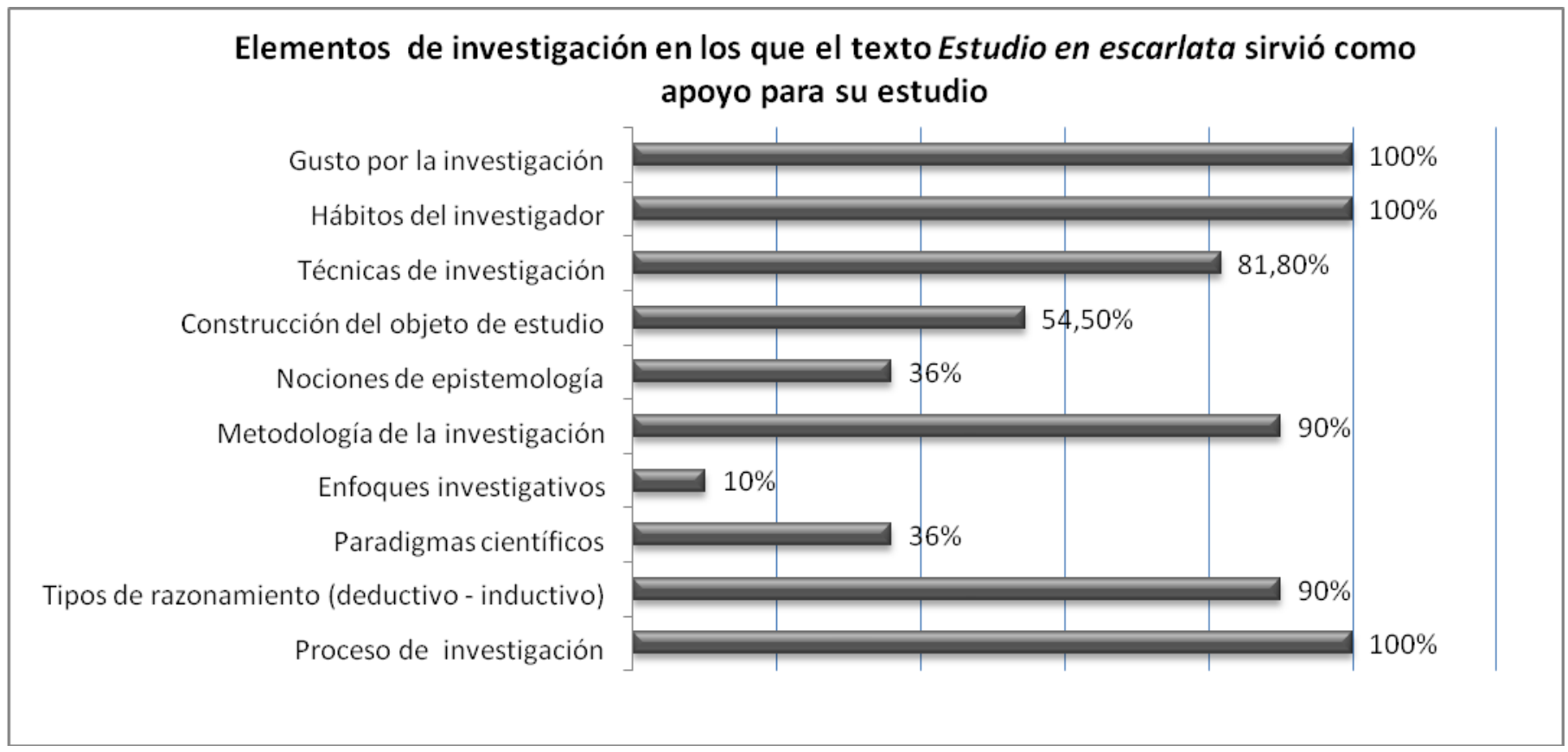

Fuente: Elaboración propia a partir de cuestionario aplicado a los estudiantes del curso FD 0135, grupo 001, II ciclo 2011 y grupo 002, I ciclo 2012.

Como se observa en el Gráfico N. 1, hay tres contenidos que indican que todos los estudiantes están de acuerdo en que la estrategia sirvió como apoyo para su estudio. Las tres temáticas son interesantes e importantes, pues de ellas depende en gran medida que cada estudiante adquiera una disposición positiva sobre la investigación. Estos contenidos son en primer lugar, el conocimiento del proceso investigativo, sobre todo, la idea de la investigación como un proceso en el que se construye conocimiento, la cual es una premisa fundamental en la investigación particularmente en la socioeducativa (todos los estudiantes están de acuerdo en que el texto favoreció el aprendizaje sobre este contenido). En segundo lugar, los hábitos y el gusto por la investigación tienen que ver con elementos que quizá no son contenidos estrictamente académicos, pero que resultan imprescindibles para el buen desarrollo de la investigación académica, pues algo fundamental para un investigador es desarrollar ciertos hábitos de trabajo constante, de indagación exhaustiva, e incluso de trabajo imaginativo y creativo, que Sherlock Holmes expone muy bien. En tercer lugar, el gusto por la investigación, lo cual permite disfrutar el trabajo que se está realizando. 
Entre el $54 \%$ y $90 \%$ de los estudiantes también estuvo de acuerdo en que el texto sirve de apoyo para el estudio de otros elementos, de relaciones con la metodología y técnicas de investigación, del tipo de razonamiento (inductivo-deductivo) y la construcción del objeto de estudio, ya que en la novela, Sherlock Holmes junto al Dr. Watson, construyen y resuelven un caso a partir de ciertas evidencias que recolectan a través de distintas técnicas y estrategias metodológicas, lo cual permite ilustrar de manera literaria y agradable, el proceso de investigación, que está constituido, parafraseando a los autores de El Oficio del Sociólogo, por la conquista, construcción, y problematización de los hechos (Bourdieu; Passeron; Chamboredon, 2003).

En ese sentido, la novela Estudio en escarlata, como recurso didáctico para la enseñanza de la investigación, resulta de gran provecho para la mediación pedagógica de distintos contenidos que normalmente son estudiados academicistamente, sin tener en cuenta que, a pesar de la gran importancia del componente académico, la investigación va más allá, implicando cuestiones de gusto, hábitos y capacidad imaginativa para establecer relaciones que se construyen y que no están dadas, contra la ilusión del saber espontáneo, sobre todo en las ciencias sociales; idea que planteaba con elocuencia Gastón Bachelard (1979): "Para un espíritu científico todo conocimiento es una respuesta a una pregunta. Si no hubo pregunta, no puede haber conocimiento científico. Nada es espontáneo. Nada está dado. Todo se construye" (p. 103).

Propiciar esos elementos en un curso de investigación es fundamental para la formación de los estudiantes, con el fin de que se acerquen de manera más creativa y más constructiva a la investigación social y educativa, alejándose de los recetarios investigativos y sobre todo metodológicos que abundan y que se pueden tomar al pie de la letra, cerradamente.

En relación con los otros tres contenidos, los cuales tienen que ver con epistemología, enfoques investigativos y paradigmas científicos, son menos los estudiantes que están de acuerdo en que la novela apoyó el estudio de esos temas. Sin embargo, hay algunos que consideraron que sí, pues quizá la interpretación que hicieron y las relaciones que establecieron, les permitieron observar esos tres temas reflejados en la obra. En todo caso, estos datos sirven para tener en cuenta que se podría hacer notar con mayor énfasis, la presencia de tales temas en la novela. 


\section{Consideraciones finales}

Si bien la investigación social y educativa es una labor que posee un carácter académico fuerte y esencial, la enseñanza de esa forma de investigación no tiene necesariamente que verse envuelta en la rigidez de los cánones de la academia. Esto no significa situar la enseñanza de la investigación fuera de la discusión académica, lo cual no es posible, significa que la labor del docente de investigación puede nutrirse y valerse de muchos otros recursos, entre ellos, como lo hemos expuesto ya, el recurso literario.

La sistematización de esta estrategia didáctica para la enseñanza de la investigación, en la educación superior, a pesar de los buenos resultados que arroja la experiencia de campo y su utilización, no supone una serie de pasos que deben seguirse de manera estricta, sino que es la forma como hemos intentado disponer de manera coherente, la utilización del texto Estudio en escarlata para la enseñanza de la investigación. Existirán muchas otras formas de abordar el tema y la obra de Conan Doyle, así como la realidad es inagotable.

De esto se sigue que la utilización de este recurso puede realizarse de muy variadas maneras, lo cual queda, en buena medida, a discreción de los participantes del proceso de aprendizaje, tanto docentes como estudiantes.

Los estudiantes han tenido -y los futuros estudiantes tendrán- con la utilización de este recurso, la posibilidad de descubrir y re-descubirir parte de la obra de Arthur Conan Doyle, así como a los célebres personajes del Sherlock Holmes y el Dr. Watson, enriqueciendo su bagaje intelectual con una obra que literariamente tiene mucho valor, que constituye un clásico de la literatura universal.

Además, sobre todo, los estudiantes que participaron de la estrategia han tenido la posibilidad de ver la investigación en la literatura, así como la literatura en la investigación, mediante un proceso cuyas principales herramientas han sido el análisis, la retroalimentación y la crítica, cuyos principales objetivos han sido brindar un espacio diferente al trabajo de estudio académico, al mismo tiempo, reforzar los contenidos estudiados en clase, mediante el recurso literario.

Desde el punto de vista docente, también el espacio es sumamente enriquecedor, pues permite disfrutar de manera menos rígida el curso, pero, por encima de ello, permite observar cómo los estudiantes se están apropiando del vocabulario propio de la investigación y estableciendo relaciones entre la novela y los contenidos que están estudiando en el curso. 
En ese sentido, resulta un recurso de alto provecho para observar el progreso de los estudiantes en el dominio de ciertos conceptos y elementos básicos de la investigación socioeducativa.

Además de los elementos y conceptos propios de la investigación socioeducativa, la obra nos ha permitido trabajar y considerar algunos elementos que no siempre están presentes en los libros de epistemología de las ciencias sociales o en los manuales de metodología de la investigación social y educativa, los cuales tienen que ver con el gusto por la investigación, los hábitos del investigador y la capacidad creativa e imaginativa del investigador como una herramienta que permite establecer relaciones que no son aparentes, y que permiten construir conocimiento, con el objetivo de conocer científicamente la realidad social y vislumbrar posibilidades de incidir sobre ella.

La implementación de una obra literaria en un ambiente académico, permite combinar dos mundos que tradicionalmente, y no menos vanamente, han sido considerados como oposiciones: ciencia y literatura, o bien, el mundo de la ficción y el de la realidad, el de la sensibilidad artística y la precisión académica-científica, o como Julio Cortázar (2008) gustaba llamar, el mundo de la regularidad y el mundo de la excepción.

\section{Referencias}

Balmaceda, Sebastián y Rosas Díaz, Ricardo. (2008). Piaget, Vygotsky y Maturana: constructivismo tres voces (2a reimpr.). Buenos Aires: Aique Grupo Editor.

Bachelard, Gastón. (1979). La Formación del espíritu científico: contribución a un psicoanálisis del conocimiento objetivo (8a. ed.). México: Siglo Veintiuno Editores.

Banos, Josep-E. (2003). El valor de la literatura en la formación de los estudiantes de medicina. Educ. méd. 6(2), 93-99.

Bonorino Ramírez, Pablo Raúl. (2011). Sobre el uso de la literatura en la enseñanza del derecho. REJIE: Revista Jurídica de Investigación e Innovación Educativa, (4), 73-90.

Borges, Jorge Luis. (1998). Borges oral. Madrid: Editorial Alianza.

Borges, Jorge Luis. (2011). Miscelánea. Barcelona: Editorial Debolsillo.

Bourdieu, Pierre, Chamboredon, Jean Claude y Passeron, Jean Claude. (2003). El oficio del sociólogo. Buenos Aires: Siglo veintiuno editores. 
Cely Rodríguez, Alexander y Moreno Lache, Nubia. (2006). La literatura: una estrategia para la enseñanza y comprensión de la geografía en la escuela. GEOENSEÑNANA, 11(2), 249- 260. Universidad Pedagógica Nacional, Bogotá-Colombia.

Copi, Irving. (1984). Introducción a la lógica (27a. ed.). Buenos Aires, Arg.: Edit. Universitaria de Buenos Aires.

Cortazar, Julio. (2008). Cuentos memorables. México: Alfaguara.

Conan Doyle, Arthur. (1956). Estudio en escarlata. México, D.F.: Novaro-México.

Durkheim, Emile. (2001). Las reglas del método sociológico. México: Ediciones Coyoacán.

Eco, Umberto. (1993). ¿Cómo se hace una tesis? Técnicas y procedimientos de estudio, investigación y escritura. Barcelona: Editorial Gedisa.

Goldmann, Lucien. (1979). Epistemología de las ciencias del hombre. Buenos Aires: Paidós

Kuhn, Thomas. (2006). La estructura de las revoluciones científicas. México: Fondo de Cultura Económica.

Sierra Bravo, Restituto. (1987). Técnicas de investigación social. Teoría y ejercicios. España: Editorial Paraninfo. 